\title{
Deep inspiration breath hold reduces the mean heart dose in left breast cancer radiotherapy
}

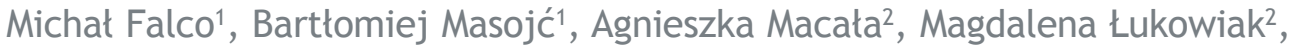 \\ Piotr Woźniak ${ }^{1}$, Julian Malicki ${ }^{3,4}$ \\ ${ }^{1}$ Radiation Oncology Department, West Pomeranian Oncology Center, Szczecin, Poland \\ ${ }^{2}$ Department of Medical Physics, West Pomeranian Oncology Center, Szczecin, Poland \\ 3 Department of Electroradiology, University of Medical Sciences, Poznan, Poland \\ ${ }^{4}$ Greater Poland Cancer Centre, Poznan, Poland
}

Radiol Oncol 2021; 55(2): 212-220.

Received 7 October 2020

Accepted 18 November 2020

Correspondence to: Michał Falco, M.D., Radiation Oncology Department, West Pomeranian Oncology Center, Strzałowska 22, 71-730 Szczecin, Poland. E-mail: mfalco@onkologia.szczecin.pl

Disclosure: No potential conflicts of interest were disclosed.

Background. Patients with left breast cancer who undergo radiotherapy have a non-negligible risk of developing radiation-induced cardiovascular disease (CVD). Cardioprotection can be achieved through better treatment planning protocols and through respiratory gating techniques, including deep inspiration breath hold (DIBH). Several dosimetric studies have shown that DIBH reduces the cardiac dose, but clinical data confirming this effect is limited. The aim of the study was to compare the mean heart dose (MHD) in patients with left breast cancer who underwent radiotherapy at our institution as we transitioned from non-gated free-breathing (FB) radiotherapy to gated radiotherapy (FB-GRT), and finally to DIBH.

Patients and methods. Retrospective study involving 2022 breast cancer patients who underwent radiotherapy at West Pomeranian Oncology Center in Szczecin from January 1, 2014 through December 31, 2017. We compared the MHD in these patients according to year of treatment and technique.

Results. Overall, the MHD for patients with left breast cancer in our cohort was $3.37 \mathrm{~Gy}$. MHD values in the patients treated with DIBH were significantly lower than in patients treated with non-gated FB (2.1 vs. $3.48 \mathrm{~Gy}, \mathrm{p}<0.0001)$ and gated FB (3.28 Gy, p < 0.0001). The lowest MHD values over the four-year period were observed in 2017, when nearly $85 \%$ of left breast cancer patients were treated with DIBH. The proportion of patients exposed to high (> 4 Gy) MHD values decreased every year, from $40 \%$ in 2014 to $7.9 \%$ in 2017, while the percentage of patients receiving DIBH increased.

Conclusions. Compared to free-breathing techniques (both gated and non-gated), DIBH reduces the mean radiation dose to the heart in patients with left breast cancer. These findings support the use of DIBH in patients with left breast cancer treated with radiotherapy.

Key words: breast cancer; gated radiotherapy; deep inspiration breath hold; free breathing gated radiotherapy; mean heart dose

\section{Introduction}

Most patients with breast cancer, who are treated surgically also undergo postoperative radiotherapy ${ }^{1,2}$, which has been shown to improve locoregional control, recurrence rates, and survival. ${ }^{3-6}$ However, long-term population-based analyses have found that postoperative radiotherapy is as- sociated with an increased risk of mortality due to radiation-induced cardiovascular disease (CVD). ${ }^{7-9}$ In recent years, better radiotherapy treatment planning protocols ${ }^{6,10,11}$ and widespread use of respiratory gating techniques - which have been applied in all modern linear accelerators - have improved cardioprotection. ${ }^{12-16}$ 
Cardioprotection can be achieved through better treatment planning protocols and respiratory gating. When this latter technique is used, the radiation is delivered only during the inspiratory phase, when the heart is at its most distant point from the chest wall, thus reducing the radiation dose to the heart. ${ }^{8,13-16}$ In recent years, the deep inspiration breath hold (DIBH) technique has become increasingly common due to the growing body of evidence showing that this approach can reduce the mean heart dose (MHD) by 1-3 Gy compared to conventional techniques. ${ }^{17}$ The use of cardioprotective techniques such as DIBH is crucial in patients with left breast cancer, as these patients have a high risk of developing heart disease within 10 years of radiotherapy treatment. ${ }^{18}$ Dosimetric studies have shown that DIBH reduces cardiac dose in comparison with free-breathing (FB) without gating. Additional data from population analyses show that MHD decreases over successive years. ${ }^{19,20}$

At our institution, we have modified the radiotherapy treatment protocols over time to reflect technological advances and a better understanding of the importance of cardioprotection. From 2014 to 2017, we gradually transitioned from treating patients with non-gated FB to gated $\mathrm{FB}$, and finally to DIBH. Although some studies have compared FB without gating to $\mathrm{DIBH}^{12-17}$, the studies analysing whether DIBH reduces the risk of cardiotoxicity in a large, real-world clinical cohort of patients are limited. ${ }^{19,20}$ Likewise, clinical data on the influence of DIBH on cardiac complications in these patients is limited.

In this context, the aim of this study was to compare differences in mean heart dose for patients with left breast cancer treated at our institution from 2014 to 2017 during which we transitioned from non-gated free-breathing (FB) radiotherapy to gated radiotherapy, and finally to DIBH.

\section{Patients and methods}

This was a retrospective analysis of all patients (n = 2022) diagnosed and treated for breast cancer at West Pomeranian Oncology Center in Szczecin from January 1, 2014 through December 31, 2017.

Patients' written inform consent about the study was waved because of retrospective clinical data analysis. The study was conducted according the Helsinki Declaration and the European Council Convention on Protection of Human Rights in BioMedicine (Oviedo 1997).
Virtually all of patients $(99.6 \%)$ received postoperative radiotherapy and 1049 (51.9\%) were treated for left breast cancer. During the study period, most patients were treated with conventional three-dimensional (3D) radiotherapy $(\mathrm{n}=$ $1513,74.8 \%$ ) or intensity-modulated radiotherapy (IMRT; $\mathrm{n}=69,3.4 \%$ ) with free-breathing. A total of 188 patients $(9.3 \%)$ underwent FB-gated radiotherapy (FB-GRT). Starting in October 2016, all new left breast cancer patients were treated with DIBH. Thus, from that point in time until the study end (2017), the DIBH technique was applied in 252 $(12.5 \%)$ patients. Gated radiotherapy during FB and DIBH were applied only to left breast cancer patients.

All patients underwent CT-based 3D planning in the therapeutic position. All patients were treated on the same linear accelerator model (Artiste, Siemens Healthcare, Erlangen, Germany). Gated radiotherapy during FB procedures was performed with assistance of a respiratory gating system (AZ733VI, Anzai Medical Co., Tokyo, Japan), which divides the normal breathing cycle into eight phases, with irradiation administered only during the inhalation phase. Patients who were able to maintain a stable breath cycle received FG-GRT if, in the clinical judgement of the treating radiation oncologist, there was a dosimetric benefit identified by any significant separation of the heart from chest wall (increase of at least $5 \mathrm{~mm}$ ). DIBH (AlignRT system Vision RT Ltd, London, UK) was used in patients expected to benefit from this approach. Patients unable to hold their breath were not considered eligible for this procedure. The DIBH irradiation technique was used with assistance of a real-time 3D surface tracking system (AlignRT) as described elsewhere. 12,21,22

Treatment planning followed institutional protocol. CTV contours were drawn according to ESTRO recommendation $\mathrm{s}^{23}$ and heart contours according to Feng et al..$^{24}$ Regional lymph nodes (ipsilateral axillary and supraclavicular ones) were irradiated in every patients with macrometastases in axillary lymph nodes as internal mammary lymph nodes (upper I-IV) in patients after mastectomy. Five millimetre margin was added to create PTV from CTV. Dose constraints for heart were $V_{20}<$ $10 \%$ (less than $10 \%$ of the organ covered be dose of 20Gy), $\mathrm{V}_{40}<5 \%$ for conventional fractionation and $\mathrm{V}_{17}<10 \%, \mathrm{~V}_{35}<5 \%$ for hypofractionated regimens.

Treatment plans were created with the Prowess Panther system for IMRT (Radiology Oncology Systems, Inc., San Diego, CA, USA) and the Oncentra Masterplan (Nucletron, Veenendaal, The 
TABLE 1. Patient clinical characteristics by year of treatment

\begin{tabular}{|c|c|c|c|c|c|c|}
\hline \multicolumn{7}{|c|}{ Variable } \\
\hline & & 2014 & 2015 & 2016 & 2017 & p-value \\
\hline \multirow{2}{*}{ Side } & Right & 155 & 277 & 278 & 263 & \multirow{2}{*}{ NS } \\
\hline & Left & 201 & 284 & 298 & 266 & \\
\hline \multirow{4}{*}{ BMI } & $<25$ & 7 & 12 & 14 & 8 & \multirow{4}{*}{ NS } \\
\hline & $25-30$ & 67 & 101 & 97 & 77 & \\
\hline & $30-35$ & 65 & 102 & 101 & 117 & \\
\hline & $>35$ & 62 & 69 & 85 & 63 & \\
\hline \multirow{2}{*}{ Operation type } & BCS & 137 & 188 & 201 & 182 & \multirow{2}{*}{ NS } \\
\hline & Mastectomy & 64 & 92 & 95 & 82 & \\
\hline \multirow{2}{*}{ Nodal status } & $N(-)$ & 129 & 159 & 180 & 174 & \multirow{2}{*}{ NS } \\
\hline & $N(+)$ & 72 & 121 & 116 & 90 & \\
\hline \multirow{2}{*}{ RNI } & No & 127 & 146 & 156 & 149 & \multirow{2}{*}{ NS } \\
\hline & Yes & 74 & 138 & 142 & 117 & \\
\hline \multirow{2}{*}{ HFX } & No & 165 & 192 & 167 & 80 & \multirow{2}{*}{$<0.0001$} \\
\hline & Yes & 36 & 92 & 131 & 186 & \\
\hline \multirow{4}{*}{$\begin{array}{l}\text { Gated } \\
\text { radiotherapy }\end{array}$} & $F B$ & 122 & 197 & 200 & 38 & \multirow{4}{*}{$<0.0001$} \\
\hline & FB-GRT & 42 & 76 & 70 & 0 & \\
\hline & $\mathrm{DIBH}$ & 0 & 0 & 24 & 228 & \\
\hline & IMRT & 37 & 11 & 4 & 0 & \\
\hline
\end{tabular}

$B C=$ breast conserving surgery; $B M I=$ body mass index; $\mathrm{DIBH}=$ deep inspiration breath hold $\mathrm{FB}=$ free breathing; $\mathrm{FB}-\mathrm{GRT}=$ free breathing gated radiotherapy; HFX = hypofractionation; IMRT = intensity-modulated radiotherapy; Nodal status = lymph node negative vs. positive: $\mathrm{NS}=$ not significant; RNI = lymph node radiotherapy; Side = indicates right vs. left location

Netherlands) for other techniques. All patients treated with FB-GRT or gated radiotherapy during FB and DIBH underwent 3D-RT. IMRT was used in patients with left breast cancer if the 3D conformal plan did not meet the prescribed dose constraints.

The data were obtained from the planning systems, which included the: MHD; the heart volume receiving $>40 \%, 60 \%, 80 \%, 100 \%$ of the defined dose $\left(\mathrm{V}_{40 \%}, \mathrm{~V}_{60 \%}, \mathrm{~V}_{80 \%}, \mathrm{~V}_{100 \%}\right)$. The MHD value was expressed in Gy and $\mathrm{V}_{40 \%}, \mathrm{~V}_{60 \%}, \mathrm{~V}_{80 \%}, \mathrm{~V}_{100 \%}$ were the value for the absolute heart volume in cubic centimetres.

The conventional dose scheme was 50 Gy (2 Gy per fraction administered daily from Monday through Friday) to the breast/chest wall, with or without nodal irradiation. A 10-16 Gy boost to the tumor bed was prescribed for patients undergoing breast-conserving surgery (BCS). Hypofractionated schemes were 42.5-45 Gy (2.25-2.5 Gy per fraction) plus a boost of $10 \mathrm{~Gy}$, or 40.05 Gy (2.67 Gy per fraction) without boost. In the subset of patients who underwent BCS, a total of 155 were given a boost dose with either intraoperative radiotherapy $(\mathrm{n}=$
93) as an early boost or brachytherapy $(n=62)$. The boost dose was not included in the present analysis. We normalized hypofractionated plans to the conventional scheme (50 Gy in 25 fractions) and recalculated them to obtain the corrected MHD $\left(\mathrm{MHD}_{\mathrm{Fx}}\right)$.

Patients who received IMRT were not included in the MHD and $\mathrm{MHD}_{\mathrm{Fx}}$ analyses, as the MHD in IMRT plans is higher than those obtained with 3D conformal radiotherapy, with a different impact on cardiac morbidity. ${ }^{20,25}$

\section{Statistical analysis}

The $\chi^{2}$ test was used to compare differences among patients treated in different years (2014 vs. 2015 vs. 2016 vs. 2017) and between radiation techniques. The level of statistical significance was set at $\mathrm{p}<$ 0.05 . Student's $t$-test was applied to assess differences between mean values (95\% confidence interval, statistical significance was set at $p<0.05)$ of MHD, $\mathrm{MHD}_{\mathrm{Fx}^{\prime}}, \mathrm{V}_{40 \%}, \mathrm{~V}_{60 \%}, \mathrm{~V}_{80 \%}, \mathrm{~V}_{100 \%}$ over time and among techniques.

\section{Results}

Table 1 shows the clinical characteristics of the left breast cancer patients and treatment parameters according to year of treatment. As that table shows, there were no significant differences in baseline characteristics of the patients (e.g., body mass index, type of surgery, axillary lymph node surgery, nodal irradiation) regardless of the year. Table 1 also shows that the use of IMRT decreased over time as the number of patients undergoing gated therapy increased. Similarly, an increasing proportion of patients received hypofractionated radiotherapy over time.

Overall, MHD values ranged from 0 to $19.44 \mathrm{~Gy}$, with a mean of 2.48 Gy (95\% confidence interval [CI], 2.39-2.57). For patients with left breast cancer, the MHD was 3.37 Gy (range, 0.56-19.44 Gy; 95\% CI 3.23-3.5) and 1.51 Gy (range 0-17.31; 95\% CI 1.43-1.58) for the right side. Overall, the $\mathrm{MHD}_{\mathrm{Fx}}$ was 2.62 (range, 0-19.44 Gy; 95\% CI, 2.53-2.71). For patients with left breast cancer, the $\mathrm{MHD}_{\mathrm{Fx}}$ was 3.52 (range, $0.66-19.44 ; 95 \%$ CI 3.38-3.65) and 1.62 Gy (range 0-17.31; 95\% CI 1.54-1.69).

Table 2 shows the MHD and $\mathrm{MHD}_{\mathrm{Fx}}$ by year of treatment, indicating that the proportion of patients with left breast cancer exposed to MHD and $\mathrm{MHD}_{\mathrm{Fx}}$ values > 4 Gy decreased every year - from $40 \%$ in 2014 to $7.9 \%$ in 2017 - with a statistically sig- 
TABLE 2. Mean heart dose $(M H D)$ and fractionation-corrected $M H D\left(M H D_{f x}\right)$ by year of treatment

\begin{tabular}{|c|c|c|c|c|c|c|}
\hline Patients, $\mathbf{n}$ & & 160 & 255 & 294 & 266 & \\
\hline \multirow{2}{*}{ MHD (Gy) } & $\geq 4$ & 62 & 80 & 83 & 18 & \\
\hline & Mean $(95 \% \mathrm{Cl})$ & $3.93(3.53-4.33)$ & $3.44(3.19-3.68)$ & $3.27(3.07-3.49)$ & $2.23(2.1-2.37)$ & \\
\hline \multirow{3}{*}{$\mathrm{MHD}_{\mathrm{fx}}(\mathrm{Gy})$} & $\geq 4$ & 64 & 87 & 89 & 21 & \\
\hline & Mean $(95 \% \mathrm{Cl})$ & $4.03(3.63-4.43)$ & $3.6(3.35-3.84)$ & $3.41(3.2-3.61)$ & $2.42(2.28-2.56)$ & \\
\hline & \multicolumn{6}{|c|}{2014 vs. 2015: $p=0.0551,2015-2016: N S, 2016-2017: p<0.0001$} \\
\hline
\end{tabular}

NS = not significant

nificant decrease in MHD values from 2014 to 2015 and from 2016 to 2017. Similarly, the maximum MHD values fell every year from 2014 to 2017, from $19.44 \mathrm{~Gy}$ to $12.27 \mathrm{~Gy}$ to 11.07 and finally to $7.36 \mathrm{~Gy}$ in 2017. Figures 1 and 2 show these results graphically, indicating an increase in the proportion and number of patients who received lower MHD $(\mathrm{p}<$ $0.0001)$ and $\mathrm{MHD}_{\mathrm{Fx}}(\mathrm{p}<0.0001)$.

Despite the above observation every year MHD and $\mathrm{MHD}_{\mathrm{Fx}}$ mean values were significantly higher for left-sided breast cancer when compared to right-sided (Table 3). Additionally Table 3 shows, that $\mathrm{MHD}$ and $\mathrm{MHD}_{\mathrm{Fx}}$ improved every year among those either irradiated to lymph nodes or not and in those with body mass index (BMI) either below or above 30. Every year patients with $\mathrm{BMI}<30$ were exposed to lower MHD and $\mathrm{MHD}_{\mathrm{Fx}}$ and in 2016 and 2017 regional nodal irradiation (RNI) led to higher $\mathrm{MHD}$ and $\mathrm{MHD}_{\mathrm{Fx}}$ values comparing to no RNI (Table 3).

As Table 4 shows, the mean $\mathrm{V}_{40 \%}, \mathrm{~V}_{60 \%}, \mathrm{~V}_{80 \%}$, and $\mathrm{V}_{100 \%}$ values all decreased year over year, although this decrease was not statistically significant every year (e.g., $\mathrm{V}_{100 \%}$ ). Notably, $\mathrm{V}_{100 \%}$ improved irrespective of the specific gating technique (FB-GRT or DIBH) versus $F B$, with the best $V_{100 \%}$ values observed in patients treated with DIBH. There was a non-significant difference in mean $\mathrm{V}_{40 \%}, \mathrm{~V}_{60 \%}, \mathrm{~V}_{80 \%}$ values when comparing gated FB-GRT to nonnon-gated FB. For all parameters $\left(\mathrm{V}_{40 \%}, \mathrm{~V}_{60 \%}, \mathrm{~V}_{80 \%}\right.$, $\mathrm{V}_{100 \%}$ ) DIBH was significantly better than gated FB-GRT. DIBH was associated with significantly lower mean $\mathrm{V}_{60 \%}$ and $\mathrm{V}_{80 \%}$ values compared to $\mathrm{FB}$. The mean $\mathrm{V}_{40 \%}$ was lower for DIBH than for $\mathrm{FB}$, but not significantly $(\mathrm{p}=0.0529)$ (Table 4$)$.

Table 5 shows the comparison according to radiation technique (FB vs. FB-GRT vs. DIBH). DIBH

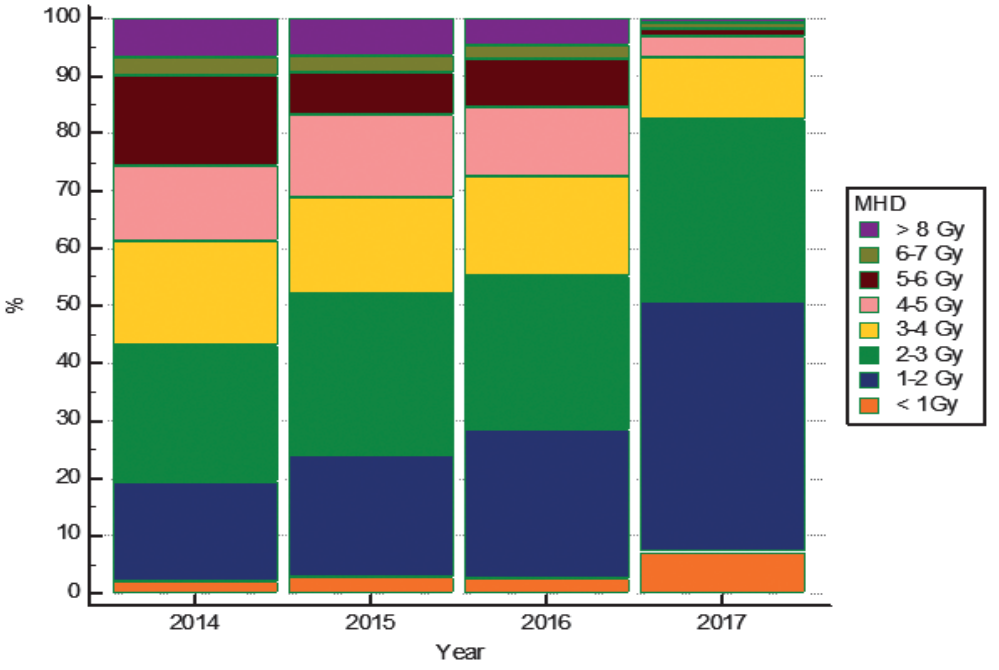

FIGURE 1. Mean heart dose (MHD) values by year 2014-2017.

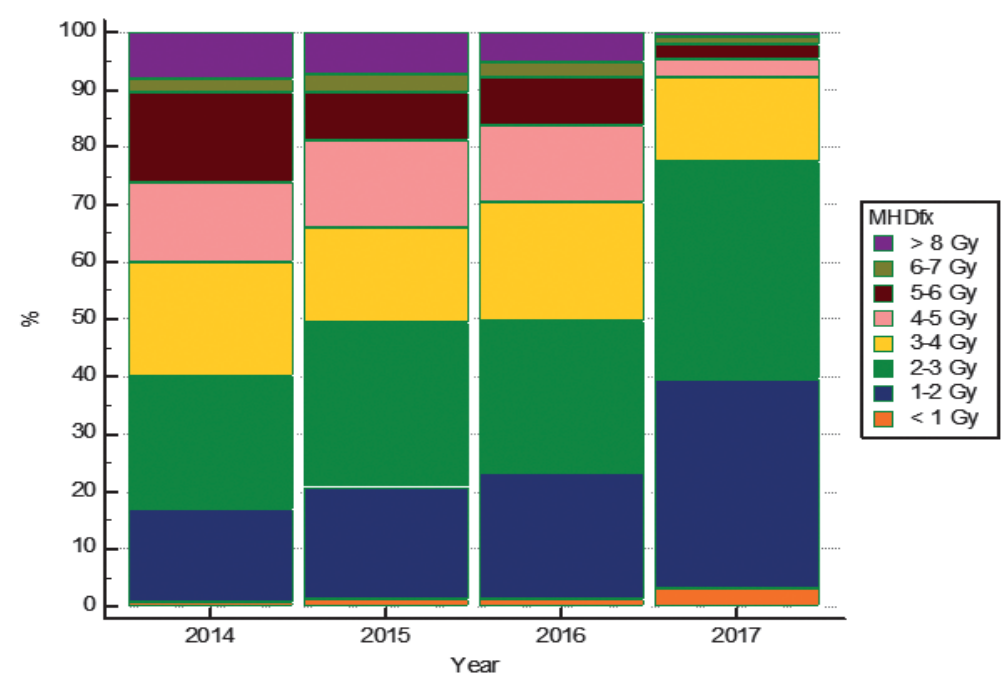

FIGURE 2. Fractionation-corrected mean heart dose $\left(M H D_{f x}\right)$ values by year 20142017. 
TABLE 3. Mean heart dose (MHD) and fractionation-corrected $M H D\left(M H D_{\mathrm{fx}}\right)$ by year of treatment and side, regional nodal irradiation and body mass index

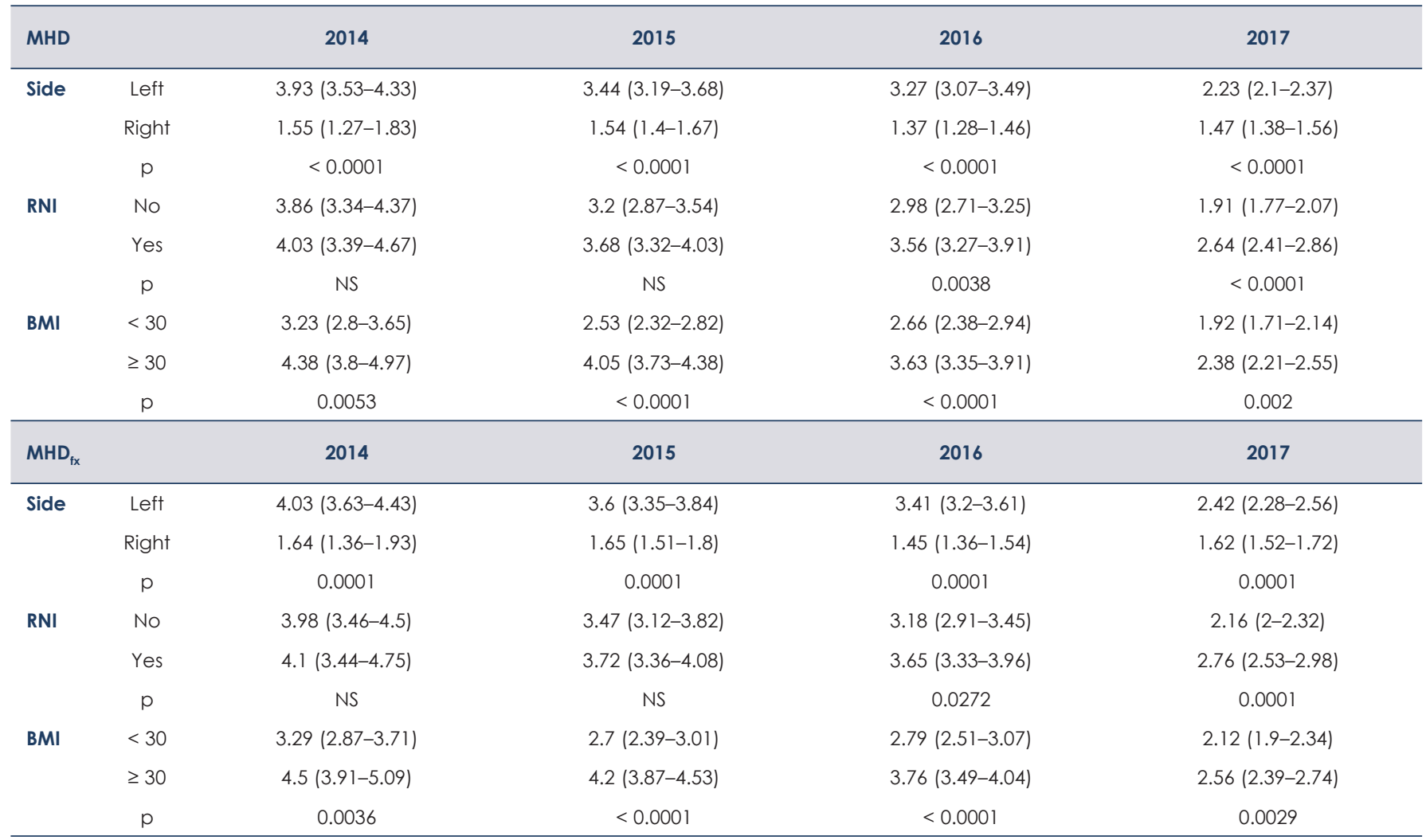

$\mathrm{BMI}=$ body mass index; NS = not significant; $\mathrm{RNI}=$ lymph node radiotherapy; Side = indicates right vs. left location;

Mean values (95\% Confidence Interval in brackets)

was associated with the lowest values $(2.1 \mathrm{~Gy}$ and 2.31 Gy, respectively), which were significantly lower than those observed for FB-GRT (3.28 Gy, p $<0.0001$ and 3.45 Gy, $p<0.0001$, respectively) and non-gated FB (3.58 Gy, p $<0.0001$ and 3.69 Gy, p < 0.0001 , respectively). There were no significant differences between the FB and FB-GRT groups. Note that fewer patients were exposed to high (> $4 \mathrm{~Gy}$ ) MHD and $\mathrm{MHD}_{\mathrm{Fx}}$ values when gated irradiation was used, particularly in the DIBH group in which only $4.8 \%$ presented a MHD $\geq 4$ Gy versus $22.4 \%$ for patients treated with FB-GRT and $35.2 \%$ in FB.

Table 6 shows no difference in MHD and $\mathrm{MHD}_{\mathrm{Fx}}$ values comparing RNI and no RNI in a group of patients without gating procedure applied. The biggest difference was observed for DIBH (MHD 2.45 vs. $1.89, \mathrm{p}<0.0001, \mathrm{MHD}_{\mathrm{Fx}} 2.58$ vs. $2.15, \mathrm{p}=$ 0.0026 respectively). MHD and $\mathrm{MHD}_{\mathrm{Fx}}$ did not differ significantly in patients with BMI below and above 30 if DIBH was used (Table 6). The difference was significant if FB and FB-GRT was used and MHD and $\mathrm{MHD}_{\mathrm{Fx}}$ were higher for patients with BMI above 30 (Table 6).

\section{Discussion}

The present study was performed to evaluate the influence of DIBH on mean heart doses in patients with left breast cancer. Overall, the MHD in patients with left breast cancer was 3.37 Gy. Patients treated with DIBH had significantly lower MHD values than patients treated with FB or FB-GRT techniques (2.1 Gy vs. 3.48 and 3.38 Gy, respectively, $\mathrm{p}<0.0001)$. The lowest MHD values were obtained in the last year of this study (2017), when nearly $85 \%$ of left breast cancer patients were treated with DIBH. Moreover, of the patients with MHD values $>4 \mathrm{~Gy}$, the smallest proportion was observed in the DIBH group. These data confirm that $\mathrm{DIBH}$ reduces the mean radiation dose to the heart in patients with left breast cancer. Drost et al. 
TABLE 4. Comparison of mean $\mathrm{V}_{40 \%}, \mathrm{~V}_{60 \%}, \mathrm{~V}_{80 \%}, \mathrm{~V}_{100 \%}$ values obtained from 2014 to 2017 and between radiation techniques

\begin{tabular}{|c|c|c|c|c|}
\hline 2014 & $0.75(0.46-1.03)$ & $7.21(5.6-8.82)$ & $16.75(13.98-19.51)$ & $33.02(28.22-37.82)$ \\
\hline 2016 & $0.20(0.02-0.38)$ & $2.73(2.04-3.42)$ & $7.91(6.56-9.26)$ & $16.9(14.49-19.30)$ \\
\hline \multirow[t]{2}{*}{2017} & $0.08(-0.0096-0.16)$ & $1.13(0.32-1.94)$ & $3.13(1.93-4.32)$ & $9.1(4.44-13.76)$ \\
\hline & 2016 vs. $2017:$ NS & 2016 vs. $2017: p=0.0029$ & 2016 vs. $2017: p<0.0001$ & 2016-2017: $p=0.0027$ \\
\hline FB & $0.37(0.22-0.52)$ & $4.46(3.67-5.25)$ & $11.2(9.9-12.5)$ & $33.13(16.22-50.03)$ \\
\hline FB-GRT & $0.76(0.36-1.17)$ & $4.7(3.61-5.79)$ & $9.87(7.83-11.9)$ & $17.75(13.95-21.56)$ \\
\hline DIBH & $0.06(-0.02-0.14)$ & $0.71(0.37-1.05)$ & $2.3(1.48-3.13)$ & $7.47(2.71-12.23)$ \\
\hline
\end{tabular}

$\mathrm{DIBH}=$ deep inspiration breath hold; $\mathrm{FB}=$ free breathing; $\mathrm{FB}-\mathrm{GRT}=$ free-breathing gated radiotherapy; $\mathrm{V}_{40 \%}, \mathrm{~V}_{60 \%}, \mathrm{~V}_{80 \%}, \mathrm{~V}_{100 \%}=$ absolute heart volume (in cubic centimetres) covered by percentage of delivered dose (40\%-100\%):

Mean values $(95 \%$ Confidence Interval in brackets)

analysed studies published between 2014 and 2017, reporting MHD 3.6 Gy in left breast cancer patients and $1.7 \mathrm{~Gy}$ if any breathing control technique was used (19). The data from 20 sites in United States show that median MHD decreased from 2.19 Gy in 2012 to 1.65 Gy in 2015 (20). Comparable values were observed for left breast cancer patients with median MHD 1.5 Gy for gated radiotherapy. ${ }^{26}$ In our study the reported values are higher. It might be explained be differences in 3D planning systems used in different centres, as mean values for right breast cancer patients in our study are twice higher than in other studies (1.51 Gy vs. $0.7 \mathrm{~Gy}){ }^{26}$ Testolin et al. presented data on 280 left breast cancer patients who underwent DIBH combined with IMRT. The mean MHD was 0.94 Gy in DIBH group and $2.14 \mathrm{~Gy}$ in those with no gating. ${ }^{27}$ Those values are lower than the ones we present, but on the other hand in mentioned trial only $11 \%$ of patients were after mastectomy and only $11.4 \%$ patients underwent RNI comparing to $44 \%$ and $31 \%$ in 2017 in our study. Nevertheless in our study mean MHD without RNI was 1.89 Gy.

There is a large body of evidence on the negative impact of excessive radiation doses to the heart. Darby et al..$^{28}$ showed that every 1 Gy increase in MHD increases the risk of CVD-related mortality by $7.4 \%$. Those authors estimated the risk of developing CVD according to increases in the MHD, as follows: $10 \%$ increased risk for MHD < 2 Gy; 30\%
TABLE 5. Mean heart dose (MHD) and fractionation corrected $M H D\left(M H D_{f x}\right)$ according to radiation technique

\begin{tabular}{|c|c|c|c|c|c|}
\hline & & FB & FB-GRT & DIBH & $p$ \\
\hline Patients, n & & 540 & 183 & 252 & \\
\hline \multirow{2}{*}{ MHD } & $<4$ Gy & 350 & 142 & 240 & $<0.0001$ \\
\hline & $\geq 4$ Gy & 190 & 41 & 12 & \\
\hline \multirow{2}{*}{$M H D_{f x}$} & $<4 \mathrm{~Gy}$ & 340 & 137 & 237 & $<0.0001$ \\
\hline & $\geq 4 \mathrm{~Gy}$ & 200 & 46 & 15 & \\
\hline
\end{tabular}

$\mathrm{DIBH}=$ deep inspiration breath hold; $F B=$ free breathing; $F B-G R T=$ free-breathing gated radiotherapy

for MHD 2-4 Gy; and 40\% for MHD at 5-9 Gy. Taylor et al. estimated that every additional $1 \mathrm{~Gy}$ in MHD is associated with a $4 \%$ increase in CVD mortality ${ }^{29}$, estimating no increase in CVD mortality risk for MHD values $<4 \mathrm{~Gy}$, but an increase in risk of up to $25 \%$ for doses ranging from 4-8 Gy.

In our cohort, $\mathrm{MHD}$ and $\mathrm{MHD}_{\mathrm{Fx}}$ values - which indicate a lower risk of CVD - trended downwards over time as radiotherapy and gating techniques improved. In 2017, most of the left breast cancer patients in our study received MHD doses below 2-4 Gy, and none were exposed to a MHD > 8 Gy. Relevantly, the only factor that changed in this period was the introduction of DIBH irradiation in October 2016. 
TABLE 6. Mean heart dose (MHD) and fractionation-corrected $M H D\left(M H D_{f x}\right)$ according to radiation technique and regional nodal irradiation, body mass index

\begin{tabular}{ccccc}
\hline MHD & & FB & FB-GRT & DIBH \\
\hline RNI & No & $3.5(3.23-3.77)$ & $3.07(2.75-3.39)$ & $1.89(1.75-2.04)$ \\
& Yes & $3.62(3.42-3.83)$ & $4.59(3-6.19)$ & $2.45(2.21-2.69)$ \\
& $\mathrm{P}$ & $\mathrm{NS}$ & 0.003 & $<0.0001$ \\
BMI & $<30$ & $2.72(2.52-2.93)$ & $2.78(2.4-3.15)$ & $1.92(1.69-2.16)$ \\
& $\geq 30$ & $4.04(2.83-4.25)$ & $3.7(3.14-4.27)$ & $2.19(2.03-2.34)$ \\
& $\mathrm{P}$ & $<0.0001$ & 0.0103 & $\mathrm{NS}$ \\
MHD $_{\text {fx }}$ & & FB & FB-GRT & DIBH \\
RNI & No & $3.71(3.44-3.99)$ & $3.25(2.94-3.57)$ & $2.15(1.99-2.3)$ \\
& Yes & $3.69(3.48-3.88)$ & $4.66(3.05-6.27)$ & $2.58(2.33-2.82)$ \\
& $\mathrm{P}$ & $\mathrm{NS}$ & 0.0061 & 0.0026 \\
BMI & $<30$ & $2.83(2.62-3.03)$ & $2.96(2.57-3.35)$ & $2.12(1.89-2.36)$ \\
& $\geq 30$ & $4.16(3.95-4.37)$ & $3.86(3.3-4.42)$ & $2.4(2.23-2.57)$ \\
& $\mathrm{P}$ & $<0.0001$ & 0.0125 & $\mathrm{NS}$ \\
\hline
\end{tabular}

$\mathrm{BMI}=$ body mass index; $\mathrm{DIBH}=$ deep inspiration breath hold; $F B=$ free breathing; $F B-G R T=$ free-breathing gated radiotherapy; NS = not significant; $\mathrm{RNI}=$ lymph node radiotherapy; Side = indicates right vs. left location:

Mean values (95\% Confidence Interval in brackets)

Sardaro et al. suggested the following planning dose constraints to achieve a low risk $(<1 \%)$ of CVD-related mortality: $\mathrm{V}_{30 \mathrm{~Gy}}<20 \mathrm{~cm}^{3}, \mathrm{~V}_{40 \mathrm{~Gy}}<10$ $\mathrm{cm}^{3}$, and $\mathrm{V}_{50 \mathrm{~Gy}}<2 \mathrm{~cm}^{3} .30$ These constraints depend only on the heart volume exposed to a given radiation dose and do not depend on the quality of organ contouring. The values reflecting those constraints in our analysis were $\mathrm{V}_{60 \%}, \mathrm{~V}_{80 \%}$, and $\mathrm{V}_{100 \%}$. In 2017, only 11 patients did not fulfil those criteria, and only 5 of those patients were treated with DIBH. In other words, $97.8 \%$ of breast cancer patients treated with DIBH radiotherapy had a less than $1 \%$ increased risk of CVD-related mortality. The largest improvement in $\mathrm{V}_{80 \%}, \mathrm{~V}_{60 \%}, \mathrm{~V}_{40 \%}$ values was observed in 2017, when DIBH was introduced. Data presented by Testolin et al. suggest that there is still possibility to improve the results as they presented mean $\mathrm{V}_{80 \%}, \mathrm{~V}_{60 \%}, \mathrm{~V}_{40 \%}$ values below $1 \mathrm{~cm}^{3}$ with combined DIBH and IMRT. ${ }^{27}$

In our cohort, gated radiotherapy during FB modestly decreased the cardiac dose compared to non-gated FB, possibly due to more stringent qualification criteria, such as the expected benefit from gated radiotherapy during $\mathrm{FB}$ and the patients' ability to cooperate with the procedure. Thus, the non-gated FB group included patients whose heart was optimally located in relation to the chest wall. However, compared to gated and non-gated FB,
DIBH resulted in significantly better heart sparing on nearly all parameters. These findings are consistent with other studies that have compared non-gated FB to $\mathrm{DIBH}$, which have shown that DIBH decreases the MHD by 33\%-66\% from the initial value compared to non-gated FB. 12-16,22,31${ }^{36}$ However, those studies are limited by the type of analyses performed: the authors created plans based on CT scans obtained during FB and DIBH, and then calculated the estimated (i.e., theoretical) benefit from gated radiotherapy techniques. By contrast, we present real-world data from routine clinical practice, confirming the findings reported by Eldredge et al. in a prospective trial that demonstrated that radiotherapy with the Active Breathing Coordinator $(A B C)$ reduced MHD values by $\geq 20 \%$ in $88 \%$ of patients. ${ }^{15}$

In $2017,86 \%$ of left side breast cancer patients successfully underwent radiotherapy with DIBH. Comparable results presented Testolin et al., but Eldredge et al. reported that $72 \%$ patients successfully underwent radiotherapy with DIBH using a different gating system (Active Breath Coordinator System, Elekta Instrument AB, Stockholm, Sweden). ${ }^{15,27}$ Surface monitoring systems seem to be more comfortable for patients.

MHD and $\mathrm{MHD}_{\mathrm{Fx}}$ were higher for RNI comparing to no RNI in those who underwent either FBGRT or DIBH. DIBH offers the best sparing in both clinical scenarios. RNI leads to higher MHD ${ }^{20,27,38}$, with the strongest impact if internal mammary lymph nodes are irradiated. ${ }^{37}$ In our study $33 \%$ of left breast cancer patients were irradiated to internal mammary lymph nodes. It may explain higher MHD values reported in our study comparing to other series. $19,20,27$

In our cohort, $\mathrm{MHD}$ and $\mathrm{MHD}_{\mathrm{Fx}}$ were higher in patients with BMI $>30$ if FB or FB-GRT was used but not DIBH. The correlation between BMI and MHD was also reported by Finazzi et al. ${ }^{37}$

\section{Study strengths and limitations}

The main limitation of this study is the retrospective design. By contrast, an important strength is the large sample size (> 1000 patients). The study clinically demonstrates that DIBH reduces the risk of cardiotoxicity versus FB and FB-GRT.

\section{Conclusions}

Our results show that the DIBH technique lowers the mean heart dose in patients with left breast can- 
cer treated with radiotherapy, minimising the risk of radiation-induced CVD. Although the clinical impact of these findings remains unknown due to the long latency period, it seems highly probable that lower radiation doses to the heart will reduce radiation-induced CVD in these patients. The data from our study, considered in the context of other published studies, suggest that DIBH should be offered to every patient with left breast cancer to reduce treatment-related morbidity and mortality.

\section{Authors' contributions and acknowledgments}

MF - study design, data collection, statistical analysis, manuscript edition; BM - study design, statistical analysis, manuscript edition; AM - data collection; MŁ - data collection; PW - manuscript edition; JM - manuscript edition.

We wish to thank Bradley Londres for professional editing of the final draft and for his invaluable suggestions to improve the text. We also thank Libby Cone from Edanz Group Japan for correcting spelling and grammar in the initial draft.

\section{References}

1. EBCTCG (Early Breast Cancer Trialists' Collaborative Group); McGale P, Taylor C, Correa C, D Cutter, F Duane, M Ewertz, et al. Effect of radiotherapy after mastectomy and axillary surgery on 10-year recurrence and 20-year breast cancer mortality: meta-analysis of individual patient data for 8135 women in 22 randomised trials. Lancet 2014; 383: 2127-35. doi: 10.1016/S01406736(14)60488-8

2. EBCTCG (Early Breast Cancer Trialists' Collaborative Group); Darby S, McGale P, Correa C, Taylor C, Arriagada R, Clarke M, et al. Effect of radiotherapy after breast-conserving surgery on 10-year recurrence and 15 -year breast cancer death: meta-analysis of individual patient data for 10,801 women in 17 randomised trials. Lancet 2011; 378: 1707-16. doi: 10.1016/ S0140-6736(11)61629-2

3. Overgaard M, Hansen PS, Overgaard J, Rose C, Andersson M, Bach F, et al. Postoperative radiotherapy in high-risk premenopausal women with breast cancer who receive adjuvant chemotherapy. Danish Breast Cancer Cooperative Group 82b Trial. N Engl J Med 1997; 337: 949-55. doi: 10.1056/ NEJM199710023371401

4. Ragaz J, Jackson SM, Le N, Plenderleith IH, Spinelli JJ, Basco VE, et al. Adjuvant radiotherapy and chemotherapy in node-positive premenopausal women with breast cancer. N Engl J Med 1997; 337: 956-62. doi: 10.1056/ NEJM199710023371402

5. Overgaard M, Jensen MB, Overgaard J, Hansen PS, Rose C, Andersson M, et al. Postoperative radiotherapy in high-risk postmenopausal breast-cancer patients given adjuvant tamoxifen: Danish Breast Cancer Cooperative Group DBCG 82c randomised trial. Lancet 1999; 353: 1641-8. doi: 10.1016/S01406736(98)09201-0

6. Cuzick J, Stewart H, Rutqvist L, Houghton J, Edwards R, Redmond C, et al. Cause-specific mortality in long-term survivors of breast cancer who participated in trials of radiotherapy. J Clin Oncol 1994; 12: 447-53. doi: 10.1200/ JCO.1994.12.3.447

7. Bouillon K, Haddy N, Delaloge S, Garbay JR, Garsi JP, Brindel P, et al. Longterm cardiovascular mortality after radiotherapy for breast cancer. J Am Coll Cardiol 2011; 57: 445-52. doi: 10.1016/j.jacc.2010.08.638
8. Darby SC, McGale P, Taylor CW, Peto R. Long-term mortality from heart disease and lung cancer after radiotherapy for early breast cancer: prospective cohort study of about 300,000 women in US SEER cancer registries. Lancet Oncol 2005; 6: 557-65. doi: 10.1016/S1470-2045(05)70251-5

9. Rehammar JC, Jensen MB, McGale P, Lorenzen EL, Taylor C, Darby SC, et al. Risk of heart disease in relation to radiotherapy and chemotherapy with anthracyclines among 19,464 breast cancer patients in Denmark, 1977-2005. Radiother Oncol 2017; 123: 299-305. doi: 10.1016/j.radonc.2017.03.012

10. Rutter CE, Chagpar AB, Evans SB. Breast cancer laterality does not influence survival in a large modern cohort: implications for radiation-related cardiac mortality. Int J Radiat Oncol Biol Phys 2014; 90: 329-34. doi: 10.1016/j. ijrobp.2014.06.030

11. Soumarová R, Rušinová L. Cardiotoxicity of breast cancer radiotherapy overview of current results. Rep Pract Oncol Radiother 2020; 25: 182-6. doi: 10.1016/j.rpor.2019.12.008

12. Czeremszyńska B, Drozda S, Górzyński M, Kępka L. Selection of patients with left breast cancer for deep-inspiration breath-hold radiotherapy technique: results of a prospective study. Rep Pract Oncol Radiother 2017; 22: 341-8. doi: 10.1016/j.rpor.2017.05.002

13. Joo JH, Kim SS, Ahn SD, Kwak J, Jeong C, Ahn SH, et al. Cardiac dose reduction during tangential breast irradiation using deep inspiration breath hold: a dose comparison study based on deformable image registration. Radiat Oncol 2015; 10: 264. doi: 10.1186/s13014-015-0573-7

14. Schönecker S, Walter F, Freislederer P, Marisch C, Scheithauer $\mathrm{H}$, Harbeck $\mathrm{N}$, et al. Treatment planning and evaluation of gated radiotherapy in left-sided breast cancer patients using the CatalystTM/SentinelTM system for deep inspiration breath-hold (DIBH). Radiat Oncol 2016; 11: 143. doi: 10.1186/ s13014-016-0716-5

15. Eldredge-Hindy $H$, Lockamy V, Crawford A, Eldredge-Hindy H, Lockamy V, Crawford A, et al. Active Breathing Coordinator reduces radiation dose to the heart and preserves local control in patients with left breast cancer: report of a prospective trial. Pract Radiat Oncol 2015; 5: 4-10. doi: 10.1016/j. prro.2014.06.004

16. Al-Hammadi N, Caparrotti P1, Naim C, Hayes J, Benson KR, Vasic A, et al. Voluntary deep inspiration breath-hold reduces the heart dose without compromising the target volume coverage during radiotherapy for left-sided breast cancer. Radiol Oncol 2018; 52: 112-20. doi: 10.1515/ raon-2018-0008

17. Smyth LM, Knight KA, Aarons YK, Wasiak J. The cardiac dose-sparing benefits of deep inspiration breath-hold in left breast irradiation: a systematic review. J Med Radiat Sci 2015; 62: 66-73. doi: 10.1002/jmrs.89

18. Boero IJ, Paravati AJ, Triplett DP, Hwang L, Matsuno RK, Gillespie EF, et al. Modern radiation therapy and cardiac outcomes in breast cancer. Int $J$ Radiat Oncol Biol Phys 2016; 94: 700-8. doi: 10.1016/j.jrobp.2015.12.018

19. Drost L, Yee C, Lam H, Zhang L, Wronski M, McCann C, et al. A systematic review of heart dose in breast radiotherapy. Clin Breast Cancer 2018; 18: e819-24. doi: 10.1016/j.clbc.2018.05.010

20. Pierce L, Feng M, Griffith KA, Jagsi R, Boike T, Dryden D, et al. Recent time trends and predictors of heart dose from breast radiation therapy in a large quality consortium of radiation oncology practices. Int J Radiat Oncol Biol Phys 2017; 99: 1154-61. doi: 10.1016/j.jjrobp.2017.07.022

21. Rong $Y$, Walston $S$, Welliver MX, Chakravarti A, Quick AM. Improving intrafractional target position accuracy using a 3D surface surrogate for left breast irradiation using the respiratory-gated deep-inspiration breath-hold technique. PLoS One 2014; 9: e97933. doi: 10.1371/journal.pone.0097933

22. Walston S, Quick AM, Kuhn K, Rong Y. Dosimetric considerations in respiratory-gated deep inspiration breath-hold for left breast irradiation. Technol Cancer Res Treat 2017; 16: 22-32. doi: 10.1177/1533034615624311

23. Offersen BV, Boersma $\sqcup$, Kirkove C, Hol S, Aznar MC, Biete Sola A, et al. ESTRO consensus guideline on target volume delineation for elective radiation therapy of early stage breast cancer. Radiother Oncol 2015; 114: 3-10. doi: 10.1016/j.radonc.2014.11.030

24. Feng M, Moran JM, Koelling T, Chughtai A, Chan JL, Freedman L, et al. Development and validation of a heart atlas to study cardiac exposure to radiation following treatment for breast cancer. Int J Radiat Oncol Biol Phys 2011; 79: 10-8. doi: 10.1016/j.ijrobp.2009.10.058

25. Fogliata A, Seppälä J, Reggiori G, Lobefalo F, Palumbo V, De Rose F, et al. Dosimetric trade-offs in breast treatment with VMAT technique. Br J Radiol 2017; 90: 20160701. doi: 10.1259/bjr.20160701 
26. Berg M, Lorenzen EL, Jensen I, Thomsen MS, Lutz CM, Refsgaard L, et al. The potential benefits from respiratory gating for breast cancer patients regarding target coverage and dose to organs at risk when applying strict dose limits to the heart: results from the DBCG HYPO trial. Acta Oncol 2018; 57: 113-9. doi: 10. 1080/ 0284186X. 2017. 1406139

27. Testolin A, Ciccarelli S, Vidano G, Avitabile R, Dusi F, Alongi F. Deep inspiration breath-hold intensity modulated radiation therapy in a large clinical series of 239 left-sided breast cancer patients: a dosimetric analysis of organs at risk doses and clinical feasibility from a single center experience. Br J Radiol 2019; 92: 20190150. doi: 10.1259/bjr.20190150

28. Darby SC, Ewertz M, McGale P, Bennet AM, Blom-Goldman U, Brønnum D, et al. Risk of ischemic heart disease in women after radiotherapy for breast cancer. N Engl J Med 2013; 368: 987-98. doi: 10.1056/NEJMoa1209825

29. Taylor C, Correa C, Duane FK, Aznar MC, Anderson SJ, Bergh J, et al. Estimating the risks of breast cancer radiotherapy: evidence from modern radiation doses to the lungs and heart and from previous randomized trials. J Clin Oncol 2017; 35: 1641-9. doi: 10.1200/JCO.2016.72.0722

30. Sardaro A, Petruzzelli MF, D'Errico MP, Grimaldi L, Pili G, Portaluri M. Radiation-induced cardiac damage in early left breast cancer patients: risk factors, biological mechanisms, radiobiology, and dosimetric constraints. Radiother Oncol 2012; 103: 133-42. doi: 10.1016/j.radonc.2012.02.008

31. Darapu A, Balakrishnan R, Sebastian P, Hussain MRK, Ravindran P, John S. Is the deep inspiration breath-hold technique superior to the free breathing technique in cardiac and lung sparing while treating both left-sided postmastectomy chest wall and supraclavicular regions? Case Rep Oncol 2017, 10: 37-51. doi: 10.1159/000453607

32. Edvardsson A, Nilsson MP, Amptoulach S, Ceberg S. Comparison of doses and NTCP to risk organs with enhanced inspiration gating and free breathing for left-sided breast cancer radiotherapy using the AAA algorithm. Radiat Oncol 2015; 10: 84. doi: 10.1186/s13014-015-0375-y

33. Kügele M, Edvardsson A, Berg L, Alkner S, Andersson Ljus C, Ceberg S. Dosimetric effects of intrafractional isocenter variation during deep inspiration breath-hold for breast cancer patients using surface-guided radiotherapy. J Appl Clin Med Phys 2018; 19: 25-38. doi: 10.1002/acm2.12214

34. Swanson T, Grills IS, Ye H, Entwistle A, Teahan M, Letts N, et al. Six-year experience routinely using moderate deep inspiration breath-hold for the reduction of cardiac dose in left-sided breast irradiation for patients with early-stage or locally advanced breast cancer. Am J Clin Oncol 2013; 36: 2430. doi: 10.1097/COC.0b013e31823fe481

35. Jensen CA1, Abramova T1, Frengen J, Lund JÅ. Monitoring deep inspiration breath hold for left-sided localized breast cancer radiotherapy with an in house developed laser distance meter system. J Appl Clin Med Phys 2017; 18: 117-23. doi: 10.1002/acm2.12137

36. Zhao F, Shen J, Lu Z, Luo Y, Yao G, Bu L, et al. Abdominal DIBH reduces the cardiac dose even further: a prospective analysis. Radiat Oncol 2018; 13: 116. doi: 10.1186/s13014-018-1062-6

37. Finazzi T, Nguyen VT, Zimmermann F, Papachristofilou A. Impact of patient and treatment characteristics on heart and lung dose in adjuvant radiotherapy for left-sided breast cancer. Radiat Oncol 2019; 14: 153. doi: 10.1186/ s13014-019-1364-3 